\title{
High-risk and multiple human papillomavirus infections among married women in Can Tho, Viet Nam
}

\author{
Lan Thi Hoang Vu*
}

Introduction: The two currently licensed human papillomavirus (HPV) vaccines are highly efficacious in preventing cervical pre-cancers related to HPV 6, 11, 16 and 18. Before implementing a large-scale HPV vaccine campaign in Viet Nam, information about the prevalence of infection with the HPV vaccine types is required. This study was done in Can Tho, the province with the highest prevalence of cervical cancer in the south of Viet Nam, to explore the distribution of other high-risk types of HPV among married women in this province.

Method: The study employed a cross-sectional design with multistage sampling. A total of 1000 participants were randomly selected, interviewed and given gynaecological examinations. HPV infection status and HPV genotyping test were completed for all participants.

Results: A broad spectrum of HPV types was reported in this study. The prevalence of cases infected with HPV 16 and/ or 18 was 7\%; the prevalence of cases infected with other high-risk HPV types was 6\%. The highest prevalence for single and multiple infections, as well as for high-risk infections, was reported for the youngest age group (less than 30 years).

Discussion: While it is relevant to implement an HPV vaccine campaign in Viet Nam due to the high prevalence of infection with HPV 16 and/or 18, it is important to note that one can be infected with multiple types of HPV. Vaccination does not protect against all types of high-risk HPV. Future vaccine campaigns should openly disclose this information to women receiving vaccines.

C ancer of the cervix is the second most common cancer in women worldwide with about 500000 new cases and 250000 deaths each year. ${ }^{1}$ Almost $80 \%$ of cases occur in low-income countries where cervical cancer is the most common cancer in women. Previous studies attributed the large decline of cervical cancer incidence in developed countries to their screening programmes and suggested that the high rate of cervical cancer mortality and morbidity in developing countries was due to ineffective or no screening programmes. ${ }^{2}$ In 2010, Viet Nam had a total of 5644 cervical cancer cases (prevalence of 13.6 per 100000 women). $^{3}$

However, the prevalence rate in the south of Viet Nam is much higher, at about 26 per 100000 women. ${ }^{3,4}$ The steadily increasing rate of this cancer in some provinces in the south has been observed in recent years. For instance, the crude rate of cervical cancer in Can Tho was only 15.7 per 100000 in 2000 but increased to 25.7 per 100000 in $2009 .^{3}$
Studies have shown that infection with highrisk human papillomavirus (HPV) can lead to cervical cancer. $^{5,6}$ Specifically, high-risk HPV types are detected in $99 \%$ of cervical cancers, and worldwide approximately $70 \%$ of cervical cancers are due to HPV types 16 and $18 .^{7-10}$ In developed countries such as the United States of America, vaccines against HPV were recommended for routine use in females aged 11 to 12 years. ${ }^{11}$ In Viet Nam, HPV vaccines have been offered since 2006. These vaccines are expensive for developing countries (US\$ 80 per dose with three doses required). These vaccines, however, prevent only four HPV types, two high-risk types (HPV 16, 18) and two low-risk types (HPV 6, 11). ${ }^{11,12}$

Before implementing a large-scale HPV vaccine campaign in the south of Viet Nam, updated data on the prevalence and distribution of the vaccine types of HPV among women is required. During 2010 and 2011, a large-scale study was done in five provinces (Ha Noi, Ho Chi Minh City, Hue, Thai Nguyen and Can Tho) to 
explore the prevalence of HPV infection and its risk factors. ${ }^{13-15}$ The prevalence of cervical HPV infection ranged from $6.1 \%$ in $\mathrm{Ha}$ Noi to $10.2 \%$ in Can Tho. Most of the positive cases were infected with high-risk HPV, especially in Ha Noi and Can Tho where more than $90 \%$ positive cases were high-risk HPV. Furthermore, in Can Tho, more than $60 \%$ of women were infected with multiple HPV types. ${ }^{15}$

This study provides additional data to explore the distribution of HPV among married women in Can Tho in order to provide more detailed information for the cervical cancer prevention programme in this province.

\section{METHOD}

\section{Study setting}

Can Tho is located on the south bank of the Hau River, a major branch of the Mekong River. It is $169 \mathrm{~km}$ from Ho Chí Minh City, Viet Nam's largest city. Can Tho is the fourth largest city in Viet Nam and the biggest city in the Mekong Delta with an estimated population of 1187089 . The majority of the population is living in urban areas (66\%). The city is divided into nine districts and 85 communes. After 120 years of development, the city now is the delta's most important centre of economics, culture, science and technology.

\section{Study population and enrolment}

This study applied a cross-sectional design and multistage sampling as described elsewhere. ${ }^{13-15}$ The estimated sample size was 1050 women using a formula for sample size for a proportion estimate with relative precision with the following parameters: anticipated prevalence of HPV as $10 \%$, relative precision of 0.25 , design effect of 1.6 and estimated non-respondent rate of $10 \%$. In the first stage, 21 communes were randomly selected from 85 communes in Can Tho. In the second stage, in each commune, 50 married women aged 18-65 were randomly selected from the list provided by the local women's union (this list contains the names of women currently living in the commune). Following an explanation about the objectives of the study, a written consent form for participation in the study was completed. The response rate was high (95.2\%) and the final sample size was 1000 women. The main reason women declined to participate was that they were uncomfortable with pelvic examination.

Information and specimen collection used the following steps. First, a personal interview was done to collect information on socio-demographic variables, obstetric/gynaecologic history and sexual lifestyle. After the interview, each participant was scheduled for a pelvic examination carried out by a gynaecologist. Samples of exfoliated cells from the ectocervix were collected with two wooden Ayre spatulas and were sent to the laboratory of the Viet Nam National Institute of Dermatology for HPV testing on the same day as the sample collections. The HPV genotyping protocol was described elsewhere. ${ }^{13}$ The protocol was reviewed and cleared by the $\mathrm{Ha}$ Noi School of Public Health Institutional Review Board (Ethical Approval Number 013/2010/YTCC-HD3).

\section{Definition of infection with high-risk HPV types}

High-risk HPV types are those that can cause cancer and include HPV 16, 18, 31, 33, 35, 39, 45, 51, 52, 53, $56,58,59,66,68$ and 82 . The low-risk group includes HPV 6, 11, 42, 43, 61, 70, 71 and 81. The current vaccines can prevent only high-risk types 16 and 18 , and low-risk types 6 and 11. Infection with HPV 16/18 in this study refers to cases infected with either HPV 16 or HPV 18 or with both of these types.

\section{RESULTS}

\section{Characteristics of population}

Most women who participated in this study were aged from 30 to 49 years (64\%). A total of 30 women (3\%) reported a history of having been diagnosed with sexual transmitted diseases, with 5\% reporting having more than two sexual partners within the last 24 months (Table 1).

\section{Single and multiple HPV infection}

Most infected cases in this study had infections with multiple types of HPV, and this was shown for each age group (Figure 1 ). The prevalence of multiple HPV infection was highest among the youngest group at $7.4 \%$. About $60 \%$ of the cases with multiple HPV infection were infected with more than two types of HPV; 
Table 1. Demographical information of the study sample, Can Tho, Viet Nam, 2010-2011

\begin{tabular}{llrr}
\hline Characteristics & & \multicolumn{2}{c}{ Frequency } \\
& $<30$ & $n$ & $\%$ \\
\hline \multirow{3}{*}{ Age group } & $30-39$ & 121 & 12.1 \\
& $40-49$ & 325 & 32.5 \\
& $>50$ & 318 & 31.8 \\
& Primary & 237 & 23.7 \\
\hline \multirow{3}{*}{ Highest education attained } & Secondary & 287 & 28.7 \\
& High school & 359 & 35.9 \\
& Higher than high school & 200 & 20.0 \\
& Government officers & 155 & 15.5 \\
\hline \multirow{3}{*}{ Occupation } & Workers/handicraft & 162 & 16.2 \\
& Small trade & 83 & 8.3 \\
& Unemployed housewife/retired & 225 & 22.5 \\
& Other & 334 & 33.4 \\
\hline History of sexual transmitted & No & 197 & 19.7 \\
\hline diseases & Yes & 970 & 97.0 \\
\hline Number of sexual partner within & 1 sexual partner & 30 & 3.0 \\
\hline the last 24 months & 2 or more & 950 & 95.0 \\
\hline & & 50 & 5.0 \\
\hline
\end{tabular}

Figure 1. Single and multiple HPV infection by age group, Can Tho, Viet Nam, 2010-2011

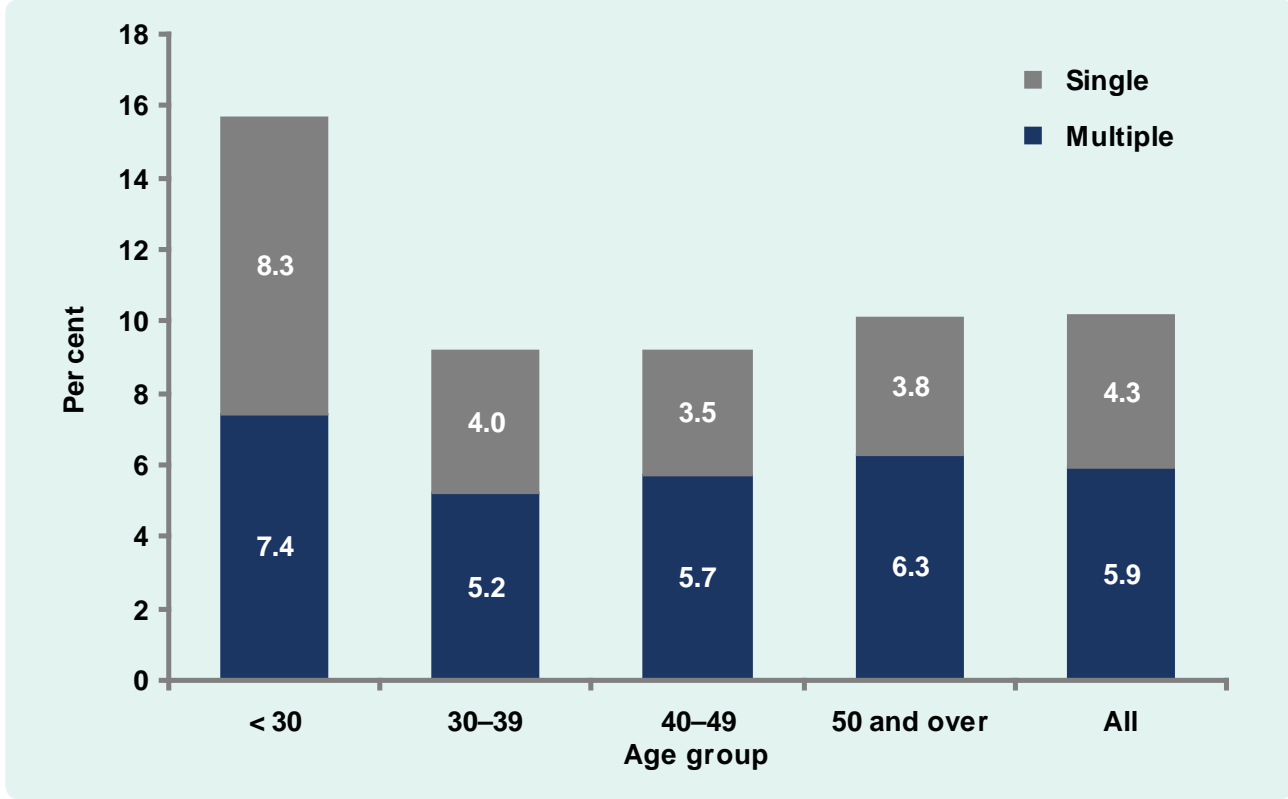

some cases were even infected with five or six different HPV types.

\section{HPV16/18 and other high-risk HPV infections}

In total, $73 \%$ of the positive cases were infected with HPV type 16/18; however, more than half of these cases were also positive with other high-risk types. More importantly, $19 \%$ of positive cases were not infected with HPV16/18 but with other high-risk types
(Figure 2). Overall, the prevalence of cases infected with HPV $16 / 18$ was $7 \%$ and the prevalence of cases infected with other high-risk HPV types (including those infected with HPV $16 / 18$ in addition to other high-risk infection types) was $6 \%$ (total 58 women).

In addition to HPV 16 and 18, there were 14 other high-risk HPV types identified in this study (Table 2). HPV 58 and HPV 52 were the most common of these high-risk types found in Can Tho, 
Figure 2. Distribution of infection with HPV $16 / 18$ and other HPV types, Can Tho, Viet Nam, 2010-2011

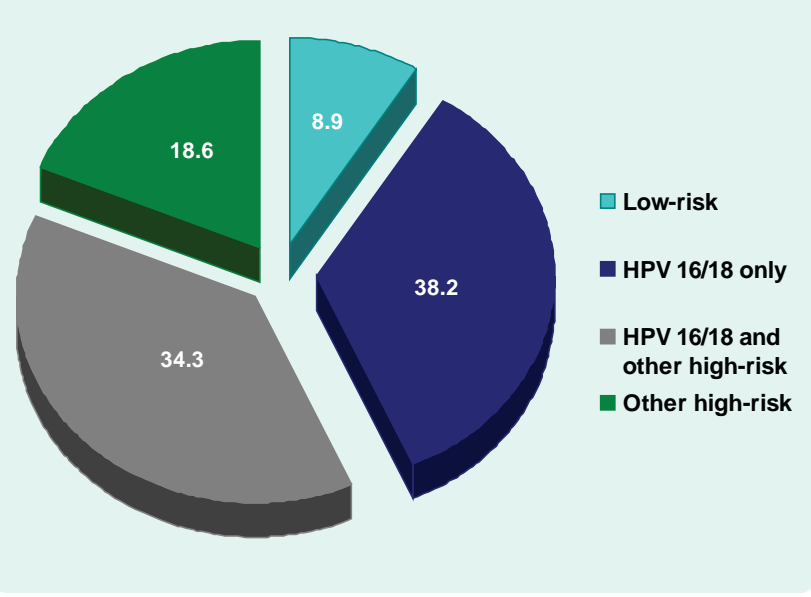

accounting for $22 \%$ and $13 \%$ of the positive cases, respectively.

\section{Infection with high-risk/low-risk HPV by age group}

Analysis by age group showed that (1) in all age groups, most cases were infected with high-risk HPV, which can lead to cancer; (2) the overall prevalence of HPV infection (i.e. including both low-risk/high-risk) was highest among the youngest group (i.e. less than
Table 2. Other types of high-risk HPV, Can Tho, Viet Nam, 2010-2011

\begin{tabular}{cccc}
\hline HPV type & $\begin{array}{c}\text { Positive } \\
\text { cases }\end{array}$ & $\begin{array}{c}\text { \% among } \\
\text { positive }\end{array}$ & $\begin{array}{c}\text { \% among } \\
\text { sample }\end{array}$ \\
\hline HPV58 & 22 & 21.6 & 2.2 \\
HPV52 & 13 & 12.7 & 1.3 \\
HPV35 & 8 & 7.8 & 0.8 \\
HPV45 & 6 & 5.9 & 0.6 \\
HPV33 & 5 & 4.9 & 0.5 \\
HPV31 & 3 & 2.9 & 0.3 \\
HPV51 & 3 & 2.9 & 0.3 \\
HPV56 & 3 & 2.9 & 0.3 \\
HPV61 & 3 & 2.9 & 0.3 \\
HPV53 & 2 & 1.9 & 0.2 \\
HPV59 & 2 & 1.9 & 0.2 \\
HPV62 & 2 & 1.9 & 0.2 \\
HPV66 & 2 & 1.9 & 0.2 \\
HPV68 & 2 & 1.9 & 0.2 \\
\hline
\end{tabular}

30-years-old); and (3) the prevalence of infection with high-risk HPV was also highest among the youngest group (Figure 3).

\section{DISCUSSION}

A broad spectrum of HPV types was reported in this study. The prevalence of cases infected with HPV 16/18 was $7 \%$ and the prevalence of cases infected with other high-risk HPV types was $6 \%$. The most common types of HPV infection were HPV 16 and 18 , similar to

Figure 3. Single and multiple HPV infection by age group, Can Tho, Viet Nam, 2010-2011

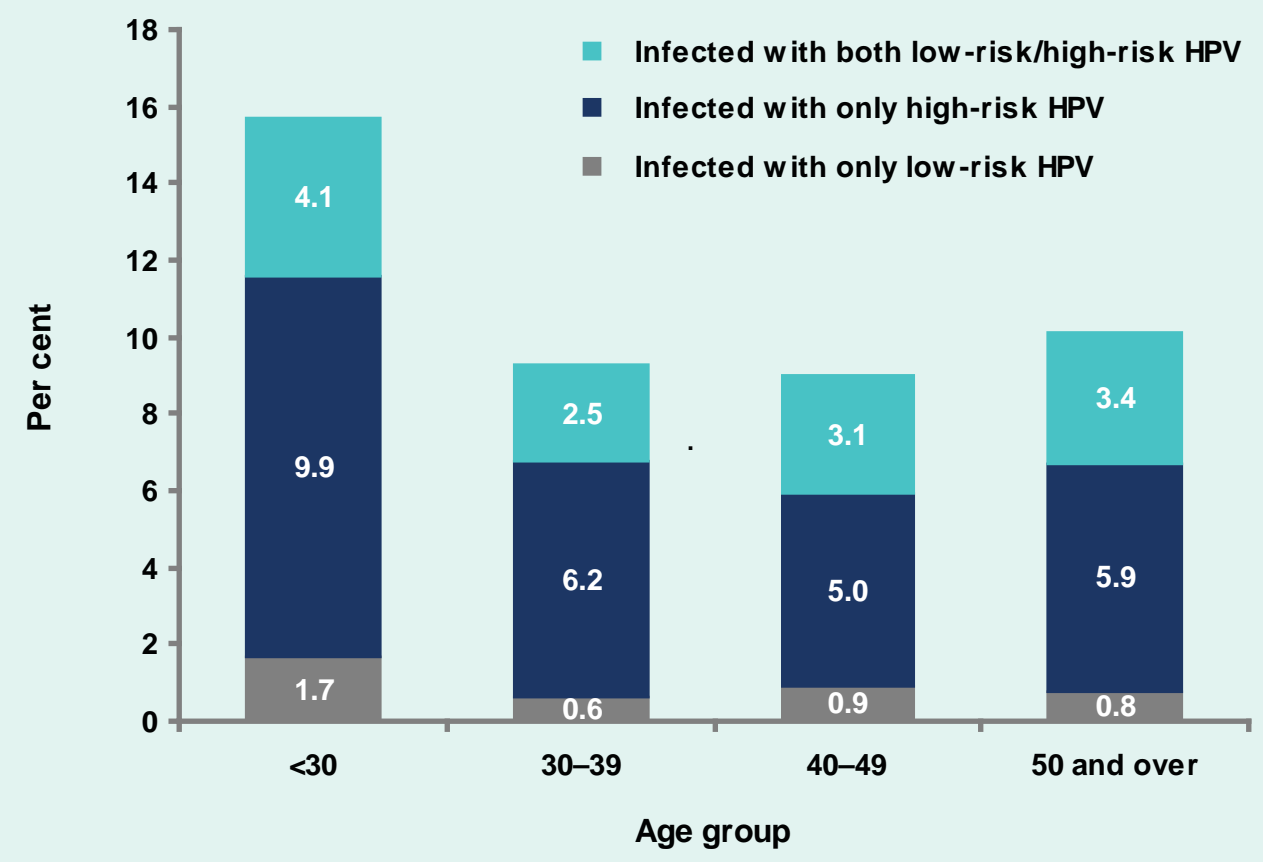


previous studies in Viet Nam and other countries. ${ }^{16-18}$ HPV 58 was also found to be a common type among women in Can Tho, as reported in China, Thailand and The Philippines. ${ }^{6}$ The higher prevalence reported for Can Tho is consistent with the higher rate of cervical cancer in the southern compared to the northern provinces of Viet Nam. Previous studies had reported that the higher rate of cervical cancer in southern Viet Nam could be attributed to the ground combat militarization of South Viet Nam during the period 1955-1975. ${ }^{19}$

Similar to a previous study, ${ }^{14}$ the prevalence of overall HPV infection as well as the prevalence of highrisk HPV infection was highest among the youngest group (aged less than 30-years-old). This result demonstrates the increasing trend of HPV infection among the younger generation in Viet Nam as well as the need for effective cervical cancer programmes. A recent study suggested that the best control programme for cervical cancer in Viet Nam is to offer HPV vaccine to young girls and screening to older women. An $70 \%$ vaccination and screening coverage rate would reduce the lifetime risk of cancer by $20.4 \%$ to $76.1 \%{ }^{20}$

Some health organizations in Viet Nam are considering two types of HPV vaccines licensed by the Food and Drug Administration (i.e. Cervarix made by GlaxoSmithKline and Gardasil made by Merck). ${ }^{12}$ Cervarix protects against only HPV types 16 and 18 , while Gardasil also protects against HPV types 6 and 11. It is important to note that one woman may be infected with multiple types of HPV at once. While $73 \%$ of the positive cases were infected with HPV $16 / 18$, more than half of these were also infected with other high-risk HPV types that may also lead to cervical cancer and which cannot be prevented by currently available vaccines. Although it is relevant to implement an HPV vaccine campaign in Viet Nam due to the high prevalence of infection with HPV 16/18 in Can Tho, it is also important to inform the women who receive the vaccines that they are not protected against all high-risk HPV types and that they still need cervical cancer screening.

In developed countries, routine Pap smear and HPV tests are recommended for screening for cervical cancer. The Pap test is a method of examining cells from the cervix and is suggested every one to two years for most women aged 21 to 29 and every two to three years for most women aged 30 or older. For women aged 30 or over, HPV tests can be done in addition to Pap smear tests. ${ }^{21}$ As with other developing countries, Viet Nam has not yet established a national policy and guidelines on cervical cancer screening.

Strict protocols to avoid biases were followed in this study: women were randomly chosen, all clinical examination and specimen collections were done by qualified gynaecologists and all samples were examined by a nationally qualified laboratory. The detection of HPV positivity using real-time polymerase chain reaction methods and the genotyping of HPV type using reverse dot blot method in this study also provided more precise results compared to the Hybrid Capture Tube Method applied in previous studies. ${ }^{17}$ However, it is important to note that this study covered only married women aged 18-65 so the results did not cover a subgroup of the population already sexually active but not yet married. This is a limitation of the study, but under the cultural and ethical norms of Viet Nam, it is very difficult to invite unmarried women to participate in a study with pelvic examinations. Women in Can Tho are also getting married at a later age, which further restricted the involvement of younger women in the study. Since the prevalence of HPV was higher in the younger age group in this study, and this group was under-represented due to the sampling frame, the actual prevalence in Can Tho might be higher than reported.

Since the findings of this study came from one urbanized province in the south of Viet Nam, caution must be taken in generalizing these findings to the entire Viet Nam population, especially to those in rural areas. In conclusion, a high prevalence of HPV infection, especially high-risk types, was observed in this study and this was higher for younger married women. As HPV infection has a high correlation with cervical cancer, this study emphasizes the need for both primary prevention of cervical cancer with HPV vaccines as well as secondary prevention with screening. Policy-makers in Viet Nam should consider making HPV vaccines and screening for cervical cancer routine practices.

\section{Conflicts of interest}

None declared.

\section{Funding}

None. 


\section{References:}

1. Ferlay J et al. GLOBOCAN 2008, Cancer Incidence and Mortality Worldwide: IARC Cancer Base No. 10 [Internet]. Lyon, France, International Agency for Research on Cancer, 2010 (http://globocan.iarc.fr, accessed 30 December 2011).

2. Sankaranarayanan R, Budukh AM, Rajkumar R. Effective screening programmes for cervical cancer in low- and middle-income developing countries. Bulletin of the World Health Organization, 2001, 79:954-962. pmid:11693978

3. National registry system for cervical cancer in Vietnam, Statistics of provincial prevalence of cervical cancer. Ha Noi, Ministry of Health, 2007.

4. Pham THA. Cancer registration in Vietnam. Asian Pacific Journal of Cancer Prevention, 2001, 2 ARC Suppl:85-90.

5. Bosch FX et al. Epidemiology and natural history of human papillomavirus infections and type-specific implications in cervical neoplasia. Vaccine, 2008, 26(Suppl 10):K1-16. doi:10.1016/j.vaccine.2008.05.064 pmid:18847553

6. Clifford $\mathrm{GM}$ et al. Human papillomavirus types in invasive cervical cancer worldwide: a meta-analysis. British Journal of Cancer, 2003, 88:63-73. doi:10.1038/sj.bjc.6600688 pmid:12556961

7. Kjaer S. Type specific persistence of high risk human papillomavirus (HPV) as indicator of high grade cervical squamous intraepitheliallesions in young women: population based prospective follow up study. BMJ (Clinical Research Ed.), 2002, 325:1-7. doi:10.1136/bmj.325.7364.572 pmid:12098707

8. Lorincz AT et al. Human papillomavirus infection of the cervix: relative risk associations of 15 common anogenital types. Obstetrics and Gynecology, 1992, 79:328-337. doi:10.1097/ 00006250-199203000-00002 pmid:1310805

9. Sasagawa $T$ et al. High-risk and multiple human papillomavirus infections associated with cervical abnormalities in Japanese women. Cancer Epidemiology, Biomarkers \& Prevention, 2001, 10:45-52. pmid: 11205488

10. Tatti S. Epidemiology of HPV Colposcopy Management Options, 2003, 1:1-5.

11. Villa LL et al. Prophylactic quadrivalent human papillomavirus (types $6,11,16$, and 18) L1 virus-like particle vaccine in young women: a randomised double-blind placebo-controlled multicentre phase II efficacy trial. Lancet Oncology, 2005, 6:271-278. doi:10.1016/S1470-2045(05)70101-7 pmid:15863374
12. FDA News: FDA Licenses New Vaccine for Prevention of Cervical Cancer and Other Diseases in Females Caused by Human Papillomavirus. Maryland, United States Food and Drug Administration, 2006 (http://www.fda.gov/NewsEvents/ Newsroom/PressAnnouncements/2006/ucm108666.htm, accessed 25 February 2012).

13. Vu LT, Le HT. Cervical human papilloma virus infection among the general female population in Vietnam: a situation analysis. Asian Pacific Journal of Cancer Prevention, 2011, 12:561-566. pmid:21545230

14. $\mathrm{Vu} \mathrm{L}$ et al. Prevalence of cervical human papillomavirus infection among married women in Ha Noi, Vietnam, 2010. Asian Pacific Journal of Public Health, 2012, 24:385-390. doi:10.1177/1010539510393727 pmid:21362673

15. Vu LT, Bui D. Prevalence of cervical HPV infection among married women in Vietnam 2011. Asian Pacific Journal of Cancer Prevention, 2012, 13(1):37-40. doi:10.7314/ APJCP.2012.13.1.037 pmid:22502703

16. Dunne EF et al. Prevalence of HPV infection among females in the United States. JAMA: Journal of the American Medical Association, 2007, 297:813-819. doi:10.1001/jama.297.8.813 pmid: 17327523

17. Giuliano AR, Papenfuss M. Human papillomavirus infection at the United States - Mexico border: implications for cervical cancer prevention and control. Cancer Epidemiology, Biomarkers \& Prevention, 2001, 10:1129-1136. pmid:11700260

18. Pham THA et al. Human Papilloma Virus in Women in the South and the North of Vietnam. International Journal of Cancer, 2002, 104:213-220.

19. Huynh ML, Raab SS, Suba EJ. Association between war and cervical cancer among Vietnamese women. International Journal of Cancer, 2004, 110:775-777. doi:10.1002/ijc.20164 pmid:15146569

20. Kim JJ et al. Exploring the cost-effectiveness of HPV vaccination in Vietnam: insights for evidence-based cervical cancer prevention policy. Vaccine, 2008, 26:4015-4024. doi:10.1016/j. vaccine.2008.05.038 pmid:18602731

21. Moyer VA; on behalf of the U.S. Preventive Services Task Force. Screening for Cervical Cancer: U.S. Preventive Services Task Force Recommendation Statement. Annals of Internal Medicine, 2012, (Mar):14. pmid:22422943 